\title{
Influência dos agentes clareadores de baixa concentração sobre a rugosidade superficial do esmalte bovino
}

\section{Influence of low concentration bleaching agents on surface roughness of bovine enamel}

\author{
Alex Correia Vieira* \\ Adriana Castro V. Andrade* \\ Mario Cezar Oliveira* \\ lane Nery S Silva**
}

\section{Resumo}

Objetivo: avaliar a rugosidade superficial do esmalte bovino após aplicação de clareadores dentais a base de peróxido de hidrogênio, em 3 diferentes formas de apresentação e concentração. Materiais e método: utilizou-se corpos de prova obtidos a partir de 40 unidades de incisivos centrais inferiores bovinos, que foram divididos, aleatoriamente, em 4 grupos experimentais $(n=10)$ e submetidos a diferentes tratamentos clareadores da seguinte maneira: Grupo 1 (controle) - nenhum tratamento clareador; Grupo 2 - clareamento com peróxido de hidrogênio 7,5\% (Total Blanc HOME- DFL), durante 1 hora, por 14 dias; Grupo 3 - clareamento com peróxido de hidrogênio 6,5\% (3D White Whitestrips- Crest) durante 30 minutos, por 14 dias; e Grupo 4 - clareamento com peróxido de hidrogênio 7,7\% (Pretty Smile- DMC) durante 30 minutos, por 14 dias. Cada corpo de prova foi submetido à leitura da rugosidade superficial e os dados obtidos foram submetidos à análise estatística por meio do ANOVA e teste de Tukey, com nível de significância de 5\%. Resultados: todos os produtos testados aumentaram a rugosidade superficial do esmalte bovino. Conclusão: o uso do peróxido de hidrogênio em baixas concentrações, nas diferentes formas de apresentação testadas, promoveu um aumento da rugosidade superficial do esmalte de dentes bovinos, proporcional ao seu tempo de atuação.

Palavras-chave: Clareamento dental. Peróxido de Hidrogênio. Esmalte Dentário.

\section{Introdução}

Atualmente, existem diversas alternativas para o restabelecimento de um sorriso estético e harmônico, sendo os procedimentos rápidos e, minimamente, invasivos os que mais despertam interesse entre os indivíduos que buscam resultados imediatos. Nesse contexto, um dos tratamentos odontológicos estéticos mais requisitados e efetivo é o clareamento dental ${ }^{1-2}$.

$\mathrm{O}$ efeito clareador pode ser conseguido pelo paciente em consultório odontológico, em casa sob supervisão profissional ou por meio de produtos de autoaplicação (over-the-counter), método que vem se tornando cada vez mais popular. $\mathrm{O}$ fácil acesso dos pacientes aos inúmeros produtos clareadores disponíveis no mercado e o seu baixo custo justificam a popularidade deste procedimento ${ }^{3-4}$.

$\mathrm{O}$ principal agente clareador para qualquer tipo de método é o peróxido de hidrogênio $\left(\mathrm{H}_{2} \mathrm{O}_{2}\right)$ que a depender da técnica terá sua concentração e tempo de atuação utilizados de forma diferenciada ${ }^{5-6}$. A efetividade desse produto ocorre pelo seu baixo peso molecular que favorece sua difusão rápida entre os tecidos mineralizados. Sua atuação se dá pela liberação de radicais livres, como moléculas de oxigênio reativo e ânions de peróxido de hidrogênio que quebram as moléculas cromogênicas da dentina em estruturas menores. Assim, não havendo cromóforos de cadeias longas, a reflexão de luz é acentuada dando ao dente um aspecto mais claro ${ }^{7-8}$.

A alteração da cor dos dentes por meio do clareamento dental é obtida por meio de uma complexa interação físico-química entre as estruturas dentárias e os agentes clareadores ${ }^{1}$. Entretanto, esse me-

Prof.(a) Adjunto, Curso de Odontologia, Universidade Estadual de Feira de Santana, Feira de Santana, Bahia, Brasil.

Mestre em Odontologia, área de concentração Endodontia, Universidade Federal de Santa Catarina, Florianópolis, SC, Brasil. 
canismo químico não está completamente elucidado e não pode ser inteiramente controlado. O que está claro é que, os radicais livres produzidos neste processo penetram pelas regiões interprismáticas do esmalte e reagem, não apenas, com as moléculas pigmentadas, mas, também, com a matrix orgânica do esmalte. Por encontrar-se localizada nas camadas entre as zonas minerais, ao se remover a matriz orgânica desta estrutura, causa-se um aumento na irregularidade superficial deste esmalte ${ }^{9}$.

As modificações do $\mathrm{pH}$ da cavidade oral causadas pela extrema instabilidade e reatividade dos radicais livres formados a partir do peróxido de hidrogênio podem, ainda, causar inúmeros efeitos a essas estruturas quando do uso prolongado de materiais clareadores. Um importante efeito negativo advindo desse maior tempo em meio ácido é a perda de estruturas minerais da superfície dental. Um aumento na sua rugosidade superficial irá propiciar um acúmulo maior de biofilme o que contribui sobremaneira para o maior risco de cárie e de doenças periodontais ${ }^{10-15}$.

Embora sejam muitas as vantagens oferecidas pelo tratamento clareador, o uso do peróxido de hidrogênio em clareamento dental é capaz de produzir efeitos negativos tanto em tecidos moles quanto nos tecidos duros da cavidade oral. Os efeitos adversos em esmalte sugerem o aumento da porosidade, a presença de áreas de erosão e depressões, assim como, alterações morfológicas permanentes em sua superfície ${ }^{16-17}$

Diante desses achados, o estudo e a mensuração da rugosidade superficial das estruturas dentárias, após o clareamento dental, torna-se extremamente necessário para a compreensão das principais alterações em esmalte decorrentes desse processo. Portanto, este trabalho tem por objetivo avaliar a rugosidade superficial do esmalte bovino após a aplicação de clareadores a base de peróxido de hidrogênio em baixa concentração, usados para clareamento caseiro supervisionado e não supervisionado (autoaplicação). A relevância deste trabalho torna-se evidente diante da análise do efeito de produtos de autoaplicação, que são vendidos como eficazes, de fácil uso e altamente seguros, devido à sua baixa concentração, além da existência de poucos trabalhos na literatura analisando os efeitos deletérios do uso desses produtos.

\section{Materiais e método}

\section{Obtenção dos corpos de prova}

Para a realização deste estudo foram utilizados 40 unidades de incisivos centrais inferiores bovinos, extraídos imediatamente após o sacrifício desses animais e armazenados em solução fisiológica de cloreto de sódio a $0,9 \%$.

Em seguida os dentes foram limpos, removendo-se mecanicamente os debris orgânicos com posterior polimento com pontas de silicone e discos de óxido de alumínio (Shofu Dental Corporation, San Marcos, CA, USA) para se obter superfícies de esmalte uniformes, bem como, eliminar possíveis imperfeições do tecido. Após esta etapa, as coroas foram separadas das raízes por meio do uso de discos diamantados dupla face (KG Sorensen, Barueri, São Paulo, Brasil) acoplados a um motor de baixa rotação em peça reta (Kavo do Brasil Ind. E Com. Ltda, Joinville, Santa Catarina, Brasil). Assim, as raízes foram descartadas e as coroas inseridas individualmente em resina acrílica autopolimerizável (Jet, Artigos, Odontológicos Clássico Ltda, São Paulo, SP, Brasil) no interior de tubos de PVC, comercializados como bucha de redução 32x25 (Plastilit, Produtos Plásticos do Paraná Ltda, Curitiba, Paraná, Brasil). Nessa etapa, as coroas foram posicionadas durante a fase plástica de polimerização da resina, deixando exposta apenas a superfície vestibular dos dentes. Ao término da reação de polimerização, foi feito o polimento manual de cada corpo de prova com lixas d'água de carboneto de silício, granulometria 600 e 1200 (3M, Rio de Janeiro, RJ, Brasil), durante 20 segundos para cada granulação. Posteriormente, foi demarcada uma área de $8 \times 8 \mathrm{~mm}$ na superfície das coroas, com auxílio de um marcador permanente 2.0 (PILOT, São Paulo, SP, Brasil), afim de delimitar a superfície de aplicação do agente clareador e a área da leitura da rugosidade superficial.

\section{Tratamento clareador}

Dessa forma, os corpos de prova já preparados, foram, aletoriamente, divididos em 4 grupos experimentais, com 10 unidades em cada, submetidos a diferentes produtos clareadores de acordo com a Tabela 1.

Tabela 1 - Agentes clareadores utilizados em cada grupo experimental, composição e fabricante.

\begin{tabular}{|c|c|c|c|}
\hline Grupo & Agente Clareador & Composição & Fabricante \\
\hline G1 (Controle) & Nenhum & & \\
\hline G2 & $\begin{array}{l}\text { Peróxido de Hidrogênio } \\
7,5 \% \text { (Total Blanc Home) }\end{array}$ & $\begin{array}{l}\text { Peróxido de hidrogênio, fluoreto de sódio, } \\
\text { nitrato de potássio, gel tixotrópico a base de } \\
\text { carbapol, amina, glicol, sacarina, conservante } \\
\text { e água. }\end{array}$ & $\begin{array}{l}\text { DFL Indústria e Comércio S.A., } \\
\text { Rio de Janeiro, RJ, Brasil. }\end{array}$ \\
\hline G3 & $\begin{array}{l}\text { Peróxido de Hidrogênio } \\
\text { 6,5\% (3DWhiteWhitestrips } \\
\text { Advanced Vivid) }\end{array}$ & $\begin{array}{l}\text { Peróxido de hidrogênio,PVP, PEG-8. água, } \\
\text { copolímero de acrilatos, hidróxido de sódio, } \\
\text { sacarina sódica }\end{array}$ & $\begin{array}{l}\text { Crest, Cincinnati, Estados } \\
\text { Unidos. }\end{array}$ \\
\hline G4 & $\begin{array}{l}\text { Peróxido de Hidrogênio } \\
7,7 \% \text { (Pretty Smile) }\end{array}$ & $\begin{array}{l}\text { Peroxydone K-30, peroxydone K-90, Álcool } \\
\text { etílico. }\end{array}$ & $\begin{array}{l}\text { DMC Equipamentos LTDA, } \\
\text { São Carlos, SP, Brasil. }\end{array}$ \\
\hline
\end{tabular}


Os agentes clareadores foram aplicados sobre o esmalte dental na área anteriormente delimitada, segundo as recomendações do fabricante de cada produto. No grupo 2 (G2), os dentes foram submetidos ao clareamento com peróxido de hidrogênio a 7,5\% (Total Blanc Home), sendo o produto aplicado com auxílio de um microbush e mantido sobre as superfícies da amostra durante o período de 1 hora, esses passos foram repetidos durante14 dias.

No grupo 3 (G3), as unidades foram clareadas utilizando o sistema Whitestrips (Crest). A fita foi recortada em um tamanho suficiente para recobrir toda área demarcada e, então, colocada em contato com a superfície das amostras e mantida pelo tempo de 30 minutos ao dia, durante 14 dias.

Os corpos de prova do grupo 4 (G4) receberam a aplicação do sistema clareador Pretty Smile (DMC) sobre a superfície dos dentes, pelo tempo de $30 \mathrm{mi}$ nutos ao dia, durante 14 dias.

Ao término de cada sessão de clareamento, removeu-se o gel das superfícies utilizando gaze e procedeu-se a lavagem abundante em água corrente. Durante o intervalo de aplicação dos géis clareadores, todos os corpos de prova foram armazenados em solução fisiólogica.

\section{Análise da rugosidade}

Cada corpo de prova foi submetido à leitura pelo rugosímetro Surftest SJ-301 (Mitutoyo, Tokyo, Japão), para determinar a sua rugosidade superficial média. A leitura considerada foi a média aritmética (Ra) entre os picos e vales percorridos pela ponta ativa do aparelho, onde o percurso de medição foi de $4,0 \mathrm{~mm}$. Realizaram-se três leituras na superfície de cada corpo de prova: uma no sentido horizontal, outra perpendicular a primeira e uma no sentido oblíquo. As médias dos valores obtidos foram registradas, tabuladas e submetidas à análise estatística. Essa etapa da pesquisa foi realizada em parceria com a Escola Bahiana de Medicina e Saúde Pública, localizada em Salvador-BA, a qual foi palco das medições.

\section{Análise estatística}

As médias dos valores obtidos foram anotadas, tabuladas e submetidas à análise estatística por meio da análise de variância a um critério (ANOVA), em seguida ao teste de Tukey, ao nível de significância de 5\%, para comparações múltiplas.

\section{Resultados}

A Tabela 2 apresenta a comparação dos valores da rugosidade superficial média $(\mathrm{Ra})$ dos grupos testados. De acordo com os dados obtidos, a rugosidade superficial foi influenciada pelo uso dos diferentes agentes clareadores, ou seja, houve um au- mento estatisticamente significante da rugosidade superficial do esmalte de dentes bovinos em todos os grupos testes (G2, G3 e G4) com relação ao grupo controle $(\mathrm{p}<0.001)$. No entanto, levando em consideração apenas os grupos submetidos aos procedimentos clareadores, pode-se perceber diferenças significativas entre esses, sendo a rugosidade superficial média do G2 superior ao G3 e G4 ( $\mathrm{p}<0.001)$.

Tabela 2 - Rugosidade superficial média (Ra) dos grupos testados, em $\mu \mathrm{m}$, apresentadas como média e desvio padrão $(\mathrm{Pd})$.

\begin{tabular}{l|c|c}
\hline \multicolumn{1}{c|}{ Grupo } & Média (Ra) & Desvio Padrão (dp) \\
\hline G1 & $1.08 \mathrm{a}$ & 0.09 \\
G2 & $1.78 \mathrm{~b}$ & 0.10 \\
G3 & $1.58 \mathrm{c}$ & 0.08 \\
G4 & $1.59 \mathrm{c}$ & 0.12 \\
\hline
\end{tabular}

Médias seguidas de letras diferentes indicam diferença estatística significante.

\section{Discussão}

Quanto ao mecanismo de ação do gel clareador na unidade dentária não há mais discordância entre os principais autores. Acredita-se que, como a dentina e o esmalte são estruturas permeáveis ou semi-permeáveis, o peróxido de hidrogênio seja capaz de difundir-se livremente por meio dessas estruturas e alterações na superfície do esmalte dental possam ocorrer $^{16,18}$. Entretanto, os estudos disponíveis ainda não elucidaram de maneira clara quais os fatores que durante o clareamento podem inferir nas alterações morfológicas em esmalte dental e de que forma eles atuam para provocar variadas manifestações $^{19-20}$.

Dentes bovinos foram selecionados para essa pesquisa por serem de mais fácil aquisição e por possuírem superfícies mais largas e planas que os dentes humanos. A literatura já evidenciou que os agentes clareadores são capazes de afetar de forma similar tanto as superfícies dentárias humanas quanto as bovinas. Dessa forma, dentes bovinos podem substituir eficazmente os dentes humanos nas pesquisas odontológicas sendo o seu uso seguro independente das técnicas e materiais clareadores utilizados ${ }^{16,21}$.

No estudo de Lia Mondelli et al. ${ }^{2}$ (2015), ao avaliar a microdureza em esmaltes bovinos após o clareamento, os autores puderam constatar que apenas em um grupo de amostra não havia acontecido alterações nas primeiras 24 horas. Nesse estudo, esse grupo foi o que utilizou a menor concentração de peróxido de hidrogênio. Entretanto, ao se continuar o processo percebeu-se que, ao sétimo dia de clareamento contínuo, essas amostras também apresentaram uma queda significativa na microdureza do esmalte. De acordo com os resultados desse trabalho foi possível perceber que agentes clareadores mesmo em baixas concentrações são passíveis 
de promover alterações na rugosidade superficial do esmalte.

Pinto et al. ${ }^{11}$ (2004) encontraram alterações morfológicas e aumento da rugosidade superficial do esmalte após o tratamento clareador com peróxido de carbamida a $10 \%$ e $7,5 \%$. Soares et.al. ${ }^{20}$ (2013) corroboraram com esses achados quando estudaram a perda mineral e as mudanças morfofisiológicas em esmaltes de dentes bovinos após o clareamento. Ao utilizar Peróxido de Carbamida em solução a $10 \%$ os autores perceberam significantes reduções na microdureza do esmalte a partir do sétimo dia de uso, enquanto que, com o produto a $16 \%$ a diminuição da microdureza foi evidenciada já a partir da primeira sessão de clareamento. Após o décimo quarto dia de aplicação constatou-se um significativo aumento nos valores da rugosidade superficial do esmalte. Em contrapartida, Duschner et al. ${ }^{22}$ (2006), ao avaliar in vitro, os efeitos de fitas clareadoras a base de peróxido de hidrogênio à $6 \%$, $6,5 \%, 13 \%$ e $16 \%$ sobre a morfologia superficial do esmalte e da dentina, observaram que o peróxido de hidrogênio nessas concentrações não foi capaz de promover alterações significativas nos tecidos dentais mineralizados. Esses resultados contrariam os achados desta pesquisa, segundo a qualficaram claras as mudanças na rugosidade superficial de todos os grupos estudados.

Já pesquisadores como Azrak et al. ${ }^{21}(2010)$ atribuíram a frequência e ao tempo de exposição do esmalte a produtos clareadores, às desmineralizações dentárias. Esses achados poderiam explicar os resultados dessa pesquisa quando o G2, grupo em que as amostras foram submetidas ao clareamento por mais tempo, apresentou a maior rugosidade superficial dos dentes clareados ${ }^{23,17}$.

Em nosso estudo foram utilizados produtos a base de peróxido de hidrogênio em concentrações semelhantes entre si, no entanto, foi observado que o Grupo 2 (G2), submetido ao uso do peróxido de hidrogênio a 7,5\%, apresentou uma maior rugosidade superficial em relação aos demais grupos testados, com diferenças estatísticas significante. Este fato pode estar relacionado ao tempo de contato do gel com os tecidos dentários. Seguindo o protocolo de aplicação recomendado pelo fabricante, as amostras do G2 totalizaram 14 horas de contato do produto com o esmalte dentário, portanto, o dobro do período dos demais grupos. Esse maior período pode ter permitido um maior trânsito dos radicais livres entre o esmalte e a dentina produzindo efeitos deletérios sobre estas estruturas. Esse argumento está em consonância com o trabalho de Bistey et al. ${ }^{25}$ (2007) que afirmaram que as alterações em esmalte são proporcionais ao tempo de tratamento e a concentração dos produtos utilizados.

Outra variante a ser considerada, diz respeito às concentrações do $\mathrm{pH}$ ao final de cada clareamento. Segundo Trentino et al. ${ }^{23}$ (2015), há uma queda no nível do $\mathrm{pH}$ durante o procedimento de clarea- mento dos principais géis, exceto, em produtos que contêm cálcio em sua composição. Soares et al. ${ }^{17}$ (2016) corroboraram com os achados nas mudanças de concentração do $\mathrm{pH}$ no antes e depois do procedimento clareador. Porém, afirmaram não haver uma correlação direta entre os valores de $\mathrm{pH}$ e mudanças na rugosidade superficial do esmalte. Alqahtani et al. ${ }^{18}(2014)$ alertaram para a maior influência do $\mathrm{pH}$ verificar-se nos estudos in vitro devido a inexistência do efeito tampão da saliva.

É imprescindível entender ainda que, as pesquisas conduzidas in vitro possuem certas limitações que impedem obtenção de um resultado totalmente fiel ao realizado in vivo ${ }^{25}$. Isso ocorre, principalmente devido ao fato de que, em laboratório, não se pode contar com o efeito tampão da saliva, bem como, com a diluição do material clareador na saliva durante a sua aplicação. Assim, novos estudos in vivo para compreender os efeitos que os agentes clareadores causam no esmalte dental tornam-se essenciais. Só dessa forma, seria possível indicar com segurança tratamentos seguros e eficazes com o máximo de preservação aos tecidos clareados.

\section{Conclusão}

O uso do peróxido de hidrogênio em baixas concentrações, nas diferentes formas de apresentação testadas, promoveram um aumento da rugosidade superficial do esmalte de dentes bovinos.

\section{Abstract}

Objective: to assess surface roughness of bovine enamel following the application of hydrogen peroxide-based tooth bleaching agents in 3 different forms of presentation and concentration. Materials and method: specimens obtained from 40 bovine lower central incisors were used. They were randomly divided into four experimental groups $(n=10)$ and subjected to the following bleaching treatments: group 1 (control) - no bleaching treatment; group 2 - bleaching with $7.5 \%$ hydrogen peroxide (Total Blanc Home-DFL) for 1 hour over 14 days; group 3 - bleaching with $6.5 \%$ hydrogen peroxide $3 D$ White Whitestrips-Crest) for 30 minutes over 14 days; and group 4 - bleaching with $7.7 \%$ hydrogen peroxide (Pretty Smile-DMC) for 30 minutes over 14 days. All specimens were subjected to surface roughness readings and data obtained were statistically analyzed by ANOVA and Tukey's test at 5\% significance level. Results: all products tested increased the surface roughness of bovine enamel. Conclusion: the use of hydrogen peroxide at low concentrations in the different forms of presentation tested promoted increased surface roughness of bovine tooth enamel, proportional to the application time.

Keywords: Tooth bleaching. Hydrogen peroxide. Dental enamel. 


\section{Referências}

1. Kwon SR, Wertz PW. Review of the mechanism of tooth whitening. J Esthet Restor Dent 2015;27(5):240-257.

2. Lia Mondelli RF, Gabriel TRCG, Rizzante FA, Magalhães AC, Bombonatti JFS, Ishikiriama SK. Do different bleaching protocols affect the enamel microhardness. Eur J Dent 2015;9(1):25-30

3. Gerlach RW, Zhou X. Vital bleaching with whitening strips: Summary of clinical research on effectiveness and tolerability. J Contempt Dent Pract 2001;2:1-16.

4. China ALP, Souza NM, Gomes YSBL, Alexandrino LD, Silva CM. Effect of Fluoride Gels on Microhardness and Surface Roughness of Bleached Enamel. The Open Dentistry Journal 2014;8:188-193.

5. Dahl JE, Pallesen U. Tooth bleaching-a critical review of the biological aspects. Crit rev oral Bio Med 2003;14:292-304.

6. Sato C, Rodrigues FA, Garcia DM, Vidal CMP, Pashley DH, Tjaderhane L, Carrilho MR, Nascimento FD, Tersariol LS.Tooth Bleaching increases dentinal protease activity. J Dent Res 2013;92(2):187-192.

7. Seghi RR, Denry I. Effects of external bleaching on indentation and abrasion characteristics of human enamel in vitro. J Dent Res 1992;71(6):1340-4

8. Kina JF, Huac C, Riehl H, Martinez TC, Sacono NT, Ribeiro APD, Costa. CAS Response of human pulps after professional applied vital tooth bleaching. Int Endod J 2010; 43:572-580.

9. Mendonça LC, Naves LZ, Garcia LFR, Correr-sobrinho L, Soares CJ, Quagliatto PS. Permeability, roughness and topography of enamel afther bleaching: tracking channels of penetration with silver nitrate. Braz J Oral Sci 2011;10(1):1-6.

10. Baumgardner KR, Sulfaro MA. The anti-inflammatory effects of human recombinant copper-zinc superoxide dismutase on pulp inflammation. J Endod 2001;27:190-5.

11. Pinto CF, Oliveira R, Cavalli V, Giannini M. Peroxide bleaching agent effects on enamel surface microhardness, roughness and morphology. Braz Oral Res 2004;18(4):306-11.

12. Tanizawa Y. Reaction characteristics of Tooth-bleaching agent containing $\mathrm{H} 2 \mathrm{O} 2$ and NaF: in vitro study of crystal structure change in treated hydroxyapatite and chemical states of incorporated fluorine. J Cosmet Sci 2005;56(2):121-34.

13. Gursoy UK, Eren DI, Bektas OO, Hurmuzlu F, Bostanci V, Ozdemir H. effect of External tooth bleaching on dental plaque accumulation and tooth discoloration. Med Oral Patol Oral Cir Bucal 2008;13(4): E266-9.

14. Chen HP, Chang CH, Liu JK, Chuang SF, Yang JY.Effect of fluoride containing bleaching agents on enamel surface properties. J Dent 2008; 36(9):1074-7.

15. Ourique SAM, Arrais CSAG, Cassoni A, Ota- Tsuzuki C, Rodrigues JA. Effects of different concentrations of carbamide peroxide and bleaching periods on the roughness of dental ceramics. Braz Oral Res 2011;25(5):453-8.

16. Tam LE, Cho W, Wang BY, Souza G. Effect of bleaching treatment on fatigue resistance and flexuaral strength of bovine dentin. J Esthet Restor Dent 2015;27(6):374-382.

17. Soares AF, Bombonatti JF, Alencar MS, Consolmagno EC, Honório M, Lia Mondelli RF. Influence of $\mathrm{pH}$, bleaching agents, and acid etching on surface wear of bovine enamel. J Appl Oral Sci 2016.Jan-Feb; 24(1):24-30.

18. Alqahtami MQ. Tooth-bleaching procedures and their controversial effects: A literature review.The Saudi Dent J 2014;26:33-46.
19. Faraoni-Romano JJ, Silveira AG, Turssi CP, Serra MC. Bleaching agents with varying concentrations of carbide and/or hydrogen peroxides: Effect on dental microhardness and roughness. J Esthet Restor Dent 2008;20:395-404.

20. Soares DG, Ribeiro APD, Sacomo NT, Loguércio ADL, Hebling J, Costa CAS. Mineral Loss and Morphological Changes in Dental Enamel Induced by a 16\% Carbamide Peroxide Bleaching Gel. Braz Dental J 2013;24(5):517-521.

21. Azrak B, Callaway A, Kurth P, Willershausen B. Influence of Bleaching Agents on Surface Roughness of Sound or Eroded Dental Enamel Speciments. J Esthet Restor Dent 2010;22(6):391-401.

22. Duschner H, Götz H, White DJ, Kozak KM, Zoladz JR. Effects of hydrogen peroxide bleaching strips on tooth surface color, surface microhardness, surface and subsurface ultrastructure, and microchemical (Raman spectroscopic) composition. J Clin Dent 2006;17(3):72-8.

23. Trentino AC, Soares AF, Duarte MA, Ishikiriama SK, Lia Mondelli RF. Evaluation of pH Levels and Surface Roughness After Bleaching and Abrasion Tests of Eight Comercial Products. Photomed Laser Surg 2015;33(7):372-7.

24. Bistey T, Nagy IP, Simo A, Hegedus C. In vitro FT-IR study of the effects of hydrogen peroxide on superficial tooth enamel. J Dent 2007;35(4):325-30.

25. Franco LM, Machado LS, Salomão FM, Dos Santos PH, Briso ALF, Sundfeld RH. Surface effects after a combination of dental bleaching and enamel microabrasion: An in Vitro and in Situ study. Dent Mater J 2016;35(1):13-20.

\section{Endereço para correspondência:}

Mario Cezar Oliveira

Universidade Estadual de Feira de Santana - UEFS. DAS

Av. Universitária s/n, DSAU, Módulo VI

44.036-900 Feira de Santana-BA

Fone: 71-991347995

E-mail: mcezar11@gmail.com

Recebido: 18/07/2016. Aceito: 20/03/2017 